\title{
The safety and efficacy of Heparin and Nadroparin compared to placebo in acute ischemic stroke - pilot study
}

\author{
Jana Dluhaa ${ }^{a}$, Stefan Sivaka, Egon Kurca ${ }^{a}$, Robert Dusenka ${ }^{b}$, Klaudia Kalmarova ${ }^{a}$, Monika Turcanova Koprusakovaa \\ Ema Kantorova ${ }^{a}$, Vladimir Nosal ${ }^{\mathrm{a}}$
}

\begin{abstract}
Aims. This study aimed to compare the efficacy and safety of heparin and nadroparin in order to provide an additional therapeutic option for patients with acute ischemic stroke in, whom systemic thrombolysis was excluded, or thrombectomy could not be performed.

Methods. We describe a prospective randomized double-blind placebo-controlled pilot study in acute ischemic stroke. The therapeutic window was between 4.5 and $24 \mathrm{~h}$ after the onset of stroke. During the first $24 \mathrm{~h}$ of treatment, the patients divided into 3 groups received placebo, heparin or nadroparin (in therapeutic doses). During the following $48 \mathrm{~h}$, each patient received nadroparin in the therapeutic dose. $24 \mathrm{~h}$ after start of treatment they began taking $100 \mathrm{mg}$ aspirin daily. The primary safety indicator was incidence of complications such as intracerebral or systemic hemorrhage, or death. Efficacy was primarily monitored by the neurological modified Rankin Scale (mRS) at 90 days.

Results. There were no signs of intracerebral or systemic bleeding in the cohort of 87 patients. Two patients died - one $(3.7 \%)$ in the heparin and one (3.8\%) in the placebo group due to causes not connected with the treatment. There was a statistically significant difference in $\mathrm{mRS}$ on the $90^{\text {th }}$ day between the heparin and placebo groups $(21(80 \%)$ vs 13 $(50 \%), P=0.0350$ ) and between the nadroparin and placebo groups (29 (85\%) vs $13(50 \%), P=0.0031)$.
\end{abstract}

Conclusion. The results show that the treatment with heparin and nadroparin is safe and effective.

Trial registration: Trial is registered in ClinicalTrials.gov: NCT01862978.

Key words: acute ischemic stroke, nadroparin, heparin, safety, efficacy, mRS

Received: June 23, 2016; Accepted with revision: August 3, 2016; Available online: September 19, 2016

http://dx.doi.org/10.5507/bp.2016.042

${ }^{a}$ Clinic of Neurology, Jessenius Faculty of Medicine in Martin, Comenius University in Bratislava and University Hospital in Martin, Slovak Republic

${ }^{b}$ Clinic of Urology, Jessenius Faculty of Medicine in Martin, Comenius University in Bratislava and University Hospital in Martin, Slovak Republic

Corresponding author: Vladimir Nosal, e-mail:vnosal@gmail.com

\section{INTRODUCTION}

Stroke represents one of the major public health problems worldwide, particularly in industrialized countries ${ }^{1-4}$. It is a frequent cause of acute hospitalization $(150,000$ cases per year vs. 130,000 cases of myocardial infarction in France), severe morbidity (the first cause of disability, the second cause of dementia), and the third cause of overall mortality5.

Since 1995, when the results of the NINDS clinical study were published, causal therapy (intravenous thrombolysis - IVT) has been generally available. Initially IVT was administered within $3 \mathrm{~h}$ of stroke onset ${ }^{6}$. In 2009, the results of the ECASS III clinical study showed acceptable efficacy and safety of IVT within $4.5 \mathrm{~h}$ from the onset of stroke ${ }^{7}$. Moreover, currently there is an option to use mechanical thrombectomy in cases of large artery occlusion. Several publications have supported this method of treatment within $6 \mathrm{~h}$ after stroke onset ${ }^{8-10}$. According to published data as well as our everyday experience, the earlier the reperfusion treatment starts, the more effective it is and the less complications there are. On the other hand, the time is a very important limiting factor of the reperfusion therapy. From clinical practice, it is clear that most patients arrive later than $4.5 \mathrm{~h}$ after the stroke onset. Other delays are caused by poor intrahospital logistics (long door-to-needle time). Even though both of these delays can be shortened, the majority of patients still do not receive the reperfusion therapy.

Before the era of reperfusion therapy, heparin or low molecular weight heparin (LMWH) had frequently been used $^{11}$. In specific cases, such as artery dissection, cerebral vein and sinus thrombosis, or cardioembolic stroke, it used to be (and sometimes still is) considered the first choice treatment.

In general, current opinions on the use of heparin/ LMWH in acute ischemic stroke therapy are very critical. This is due especially to the results of several clinical studies, showing that there is a higher incidence of hemorrhagic complications, especially hemorrhagic transformation of ischemic tissue in patients treated with LMWH or heparin ${ }^{12-19}$. The use of heparin or LMWH is thus, according to current guidelines, accepted only as a prophylaxis for deep venous thrombosis. However, most of the published studies have not respected current views on stroke pathophysiology and treatment. Further, 
design flaws make comparison difficult. Details will be discussed later.

There is a lack of therapeutic options in the initial 4.5-24 h after stroke onset in those patients who have not undergone reperfusion therapy, and our personal positive long-term clinical experiences with heparin/LMWH treatment were the basis for our decision to start this clinical study. We have designed a prospective clinical study, with current views on stroke pathophysiology taken into the account.

The primary aim of this study (safety endpoint) is to establish whether or not patients treated with heparin or nadroparin are at higher risk of intracranial hemorrhage or major system hemorrhage compared to placebo. The other aim (efficacy endpoint) is to compare the efficacy of heparin and LMWH to placebo. Efficacy is measured by several commonly used scales - National Institutes of Health Stroke Scale (NIHSS), modified Rankin Scale (mRS), and Barthel Index (BI).

\section{METHODS}

The primary aim of our study was to test whether treatment with intravenous heparin sodium and subcutaneous nadroparin calcium in the full anticoagulant dosage administered within the first 4.5 to $24 \mathrm{~h}$ of an acute ischemic stroke is safe and effective.

Our controlled clinical trial was a single centre, prospective, randomized, double-blind, and placebo-controlled. The recruitment period was 24 months.

The study has three arms with patient randomized in the ratio $1: 1: 1$

1) Intravenous heparin sodium. Patients received heparin sodium during the first $24 \mathrm{~h}$ of treatment. After this period, they received a therapeutic dose of nadroparin calcium subcutaneously, adjusted according to body weight (Fig. 1). The nadroparin treatment lasted 48 $\mathrm{h}$. The heparin treatment included the initial bolus of $2500 \mathrm{UI}$ and further continuous application of 1000 $\mathrm{UI} / \mathrm{h}(18-20 \mathrm{UI} / \mathrm{kg} / \mathrm{h})$. aPTT was checked every $6 \mathrm{~h}$ and the dosage is adjusted in order to reach $2-2.5 \mathrm{x}$ of the initial aPTT value.

2) Subcutaneous nadroparin calcium. Nadroparin was administered subcutaneously according to body weight within $72 \mathrm{~h}$.

3) Intravenous placebo. Placebo (saline solution) was administered within the first $24 \mathrm{~h}$ by an IV pump. For the next $48 \mathrm{~h}$ patients received subcutaneous nadroparin calcium in the therapeutic dose, according to body weight.

$24 \mathrm{~h}$ after the treatment initiation a second CT scan was performed and to exclude intracranial hemorrhage, all patients receive $100 \mathrm{mg}$ of aspirin daily.

\section{Screening procedure}

Inclusion criteria: acute ischemic stroke with symptoms duration of at least $30 \mathrm{~min}$, treatment initiation with-

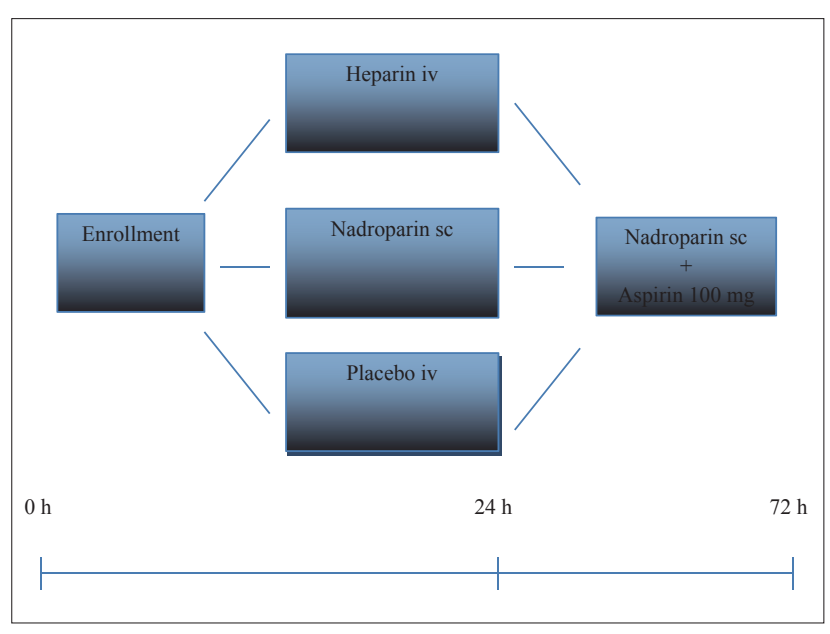

Fig. 1. Scheme of the treatment.

in 4.5 to $24 \mathrm{~h}$ after the onset of stroke, mRS (modified Rankin Scale) 0-1 (at least one month before the event), NIHSS (The National Institutes of Health Stroke Scale) $\geq 6$ and $\leq 25$, age: $18-80$ years, informed consent signed, willingness and ability to comply with the study protocol.

Exclusion criteria: intracranial hemorrhage verified by $\mathrm{CT}$, spontaneous rapid improvement of stroke symptoms before initiation of the treatment, large ischemia visible on the initial CT covering more than $2 / 3$ of the territory of the affected artery, epileptic seizure at the onset of stroke, acute ischemic stroke on initial CT, recent ischemic stroke treated with IVT or planned mechanical recanalization, stroke, myocardial infarction or serious trauma within the previous 3 months, platelet count $<100.000 / \mathrm{mm}^{3}$, refractory hypertension, systolic pressure $>185 \mathrm{mmHg}$ or diastolic pressure $>110 \mathrm{mmHg}$ despite therapy, blood glucose $<2.77$ or $>22.15 \mathrm{mmol} / \mathrm{L}$, bleeding diathesis, severe liver or renal lesion, oral or parenteral anticoagulant treatment at the time of stroke onset, current or previous life-threatening bleeding, major surgery within the previous 3 months, malignancy, active tuberculosis, pregnancy, known heparin or nadroparin allergy, alcohol and/or drug abuse, participation in another clinical study.

Safety endpoints: major bleeding (i.e. gastrointestinal bleeding), intracranial hemorrhage (intracerebral, subarachnoid), death.

Evaluation of endpoints: Safety was evaluated by an initial CT scan before randomization and another one $24 \mathrm{~h}$ later. mRS, NIHSS, and BI were performed on the $3^{\text {rd }}, 7^{\text {th }}, 30^{\text {th }}$, and $90^{\text {th }}$ day. As the efficacy endpoint of self independence, we considered an mRS score of 0-2 points on the $90^{\text {th }}$ day. We defined NIHSS score of $0-1$ points as a very good clinical neurological state (no or very mild disability), NIHSS score of 2-8 points as a good clinical state (mild to moderate disability), and NIHSS score of $\geq 9$ as a severe neurological state (severe disability), and death. BI values were divided into three categories: BI score of 95-100 - no need of assistance in daily activities, BI score of 55-90 - slight dependence, BI score of 0-50 moderate to severe dependence. 
Table 1. Time and CT characteristic of groups.

\begin{tabular}{|c|c|c|c|c|c|}
\hline $\begin{array}{l}\text { Time onset } \\
\text { of treatment }\end{array}$ & $\begin{array}{c}\text { Overall } \\
\mathrm{n}=87(\%)\end{array}$ & $\begin{array}{c}\text { Nadroparin } \\
n=34(\%)\end{array}$ & $\begin{array}{c}\text { Heparin } \\
\mathrm{n}=27(\%)\end{array}$ & $\begin{array}{c}\text { Placebo } \\
\mathrm{n}=26(\%)\end{array}$ & $\begin{array}{c}P \\
0.1075\end{array}$ \\
\hline$>4.5 \leq 12 \mathrm{~h}$ & $64(73.56)$ & $21(61.76)$ & $23(76.92)$ & $20(73.56)$ & \\
\hline$>12 \leq 24 \mathrm{~h}$ & $23(26.44)$ & $13(38.24)$ & $4(23.08)$ & $6(26.44)$ & \\
\hline $\begin{array}{l}\text { CT } 0 \mathrm{~h} \text { - on admission } \\
\text { Ischemic lesion }\end{array}$ & $23(26.44)$ & $12(35.29)$ & $5(18.52)$ & $6(23.08)$ & 0.3023 \\
\hline $\begin{array}{l}\text { CT } 24 \mathrm{~h} \text { - control } \\
\text { Ischemic lesion } \\
\text { Intracerebral hemorrhagge }\end{array}$ & $\begin{array}{c}54(62.07) \\
0(0.00)\end{array}$ & $\begin{array}{c}21(61.76) \\
0(0.00)\end{array}$ & $\begin{array}{l}6(59.26) \\
0(0.00)\end{array}$ & $\begin{array}{c}17(65.38) \\
0(0.00)\end{array}$ & 0.8988 \\
\hline
\end{tabular}

\section{Process of patient randomization and blinding}

A list of randomized numbers $(1,2,3)$ with a distribution ratio 1:1:1 was created by the EDGARII computer program. Numbers 1, 2 and 3 represent Heparin, Nadroparin, and Placebo arms, respectively. This list was open only to nurses, who prepared the medication. Patients were not aware of the type of medication they received. The clinical-state evaluator was also blinded to the therapy and results of aPTT. The Ethics Committee of Jessenius Faculty of Medicine in Martin, Comenius University in Bratislava accepted the study protocol including the informed consent.

\section{Statistical analysis}

All values are expressed as mean +/- standard deviation (SD). Shapiro-Wilk test was used to test the normality of the data. Because of non-normal data distribution, the nonparametric Kruskal-Wallis test and Man-Whitney $\mathrm{U}$ test with Bonferroni correction were used for the comparison between the groups. For comparison between paired groups, Wilcoxon signed ranks test was used. For comparision of categorical variables, the chi-square test was used. The significance level was set to $\alpha=0.05$. Statistical software STATISTICA (StatSoft) and Mathlab (Mathworks) were used for calculations.

\section{RESULTS}

The study lasted from May 2013 to February 2016. We enrolled 87 patients with acute ischemic stroke. There were 50 men $(57.47 \%)$ and 37 women (42.53\%). Patients were randomized into three treatment groups. 34 patients received nadroparin, 27 heparin and 26 patients were in the placebo group. The groups did not differ in age $(P=0.5133)$. Mean age was $70 \pm 9.3$ years. Fifty-one patients $(58.62 \%)$ were between 70 and 80 years old.

The treatment began on average $9.8 \pm 5 \mathrm{~h}$ (median: $8 \mathrm{~h}$ ) after the onset of stroke. There was no difference in the time between the three groups $(P=0.6349)$. The initial brain CT detected early signs of brain ischemia in $23(26.44 \%)$ patients and no new lesions in $64(73.56 \%)$ patients of the total cohort. The follow-up CT revealed new ischemia in 54 (62.07\%) patients and no intracranial haemorrhage in any of the patients (Table 1). The average blood pressure before the treatment was $160 \pm 22.7$ $\mathrm{mmHg}$ systole and $84 \pm 12.7 \mathrm{mmHg}$ diastole. During the first $24 \mathrm{~h}$ the average blood pressure was $150 \pm 16 \mathrm{mmHg}$ systole and $79 \pm 8.4 \mathrm{mmHg}$ diastole.

\section{Endpoints \\ Safety endpoint}

In the 87 patients, we did not detect any signs of intracerebral or systemic bleeding. Two patients died (one $(3.7 \%)$ in the heparin and one $(3.8 \%)$ in the placebo group). Overall mortality was $2.3 \%$. There was no statistically significant difference between the groups $(P=0.5180)$ in mortality.

\section{Efficacy endpoints \\ National Institute of Health Stroke Scale (NIHSS)}

The average value of NIHSS before the beginning of treatment was $7.5 \pm 2.3$ (median 7). NIHSS values of the included patients were between 6 and 17. There was no

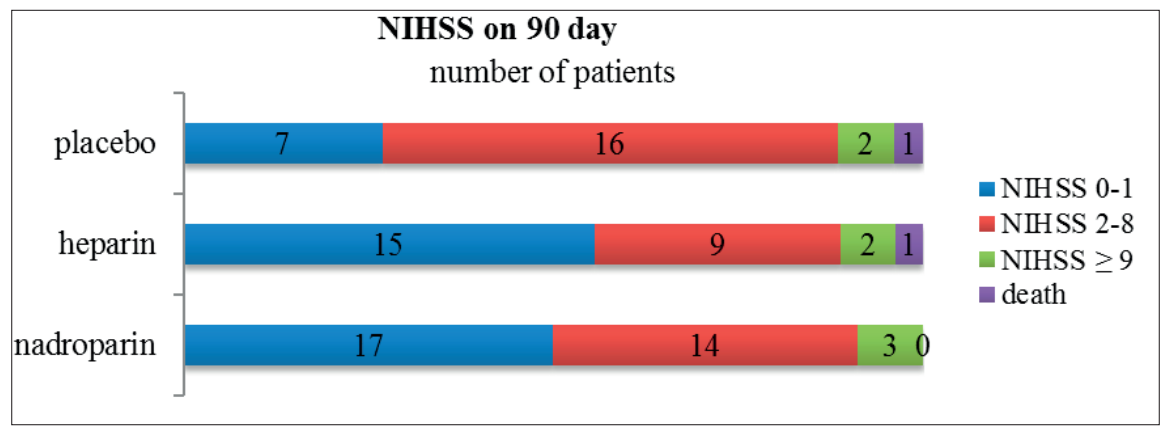

Fig. 2. NIHSS on 90th day. 
significant difference $(P=0.1130)$ between the three groups in the NIHSS scores on the $90^{\text {th }}$ day (NIHSS $0-1 ; 2-8 ; \geq 9$; death) (Fig. 2).

\section{Modified Rankin Scale (mRS)}

Self independence was evaluated by $\mathrm{mRS}$ on the $90^{\text {th }}$ day. In the total cohort the value of mRS 0-2 was reached by $63(72.41 \%)$ patients, in the nadroparin group it was $29(85 \%)$, in the heparin group it was $21(80 \%)$, and in the placebo group it was $13(50 \%)$ patients. There was a statistically significant difference between the heparin and the placebo groups ( 21 ( $80 \%)$ vs $13(50 \%), P=0.0350)$ and between the nadroparin and the placebo groups (29 ( $85 \%)$ vs 13 (50\%), $P=0.0031$ ) (Fig. 3 ).

\section{Barthel Index (BI)}

The need of assistance in daily activities was evaluated by the Barthel Index neurological scale. There was no statistically significant difference $(P=0.2029)$ between the BI groups $(95-100,5-90,0-50)$ on the $90^{\text {th }}$ day (Fig. 4$)$.

\section{DISCUSSION}

Acute ischemic stroke is a frequent and disabling disease. Despite current therapeutic approaches such as intravenous thrombolysis and mechanical thrombectomy, the majority of patients still do not receive this therapy. This is mainly caused by long delays between the onset of stroke and the beginning of therapy. The delay can be influenced (i.e. patient education, improved patient management), but only to a certain extent. Another possibility is to find new therapeutic approaches, what was the aim of our study.

In our work, we focus on the acute phase of ischemic stroke. There are three ways heparin or LMWH can be used - as a treatment in acute phase of ischemic stroke, as a secondary stroke prevention (short or long term), or as a prevention of stroke complications such as deep venous thrombosis with subsequent pulmonary embolism. ASA/AHA or ESO stroke guidelines do not recommend heparin or its derivates for the treatment of acute ischemic stroke ${ }^{20-21}$. These recommendations reflect the results of large clinical studies and meta-analyses ${ }^{12,17,19}$. Unfortunately, in most published clinical studies, heparin or LMWH were used later than $24 \mathrm{~h}$ from the onset of a stroke. There are only a few clinical studies, where the treatment is initiated within the first $24 \mathrm{~h}^{14,22}$.

One of the largest randomized studies regarding safety and efficacy of heparin was the International Stroke Trial (IST) with 19435 patients enrolled. The results showed, that there were significantly more deaths due to hemorrhagic stroke ( 28 vs $15, P<0.05$ ) or extracranial hemorrhage ( 12 vs $3, P<0.05)$ compared to non-heparin group within 14 days from the treatment initiation. On the other hand, heparin treated patients had significantly fewer recurrent ischemic strokes within 14 days (283 vs 370 , $P<0.01)$. Among pooled fatal and non-fatal events, there was a significant increase in hemorrhagic strokes (120 vs $41, P<0.00001)$ and extracranial hemorrhages (129 vs $37, P<0.00001)$. However, careful analysis can reveal significant deficiencies in the study design. It is possible

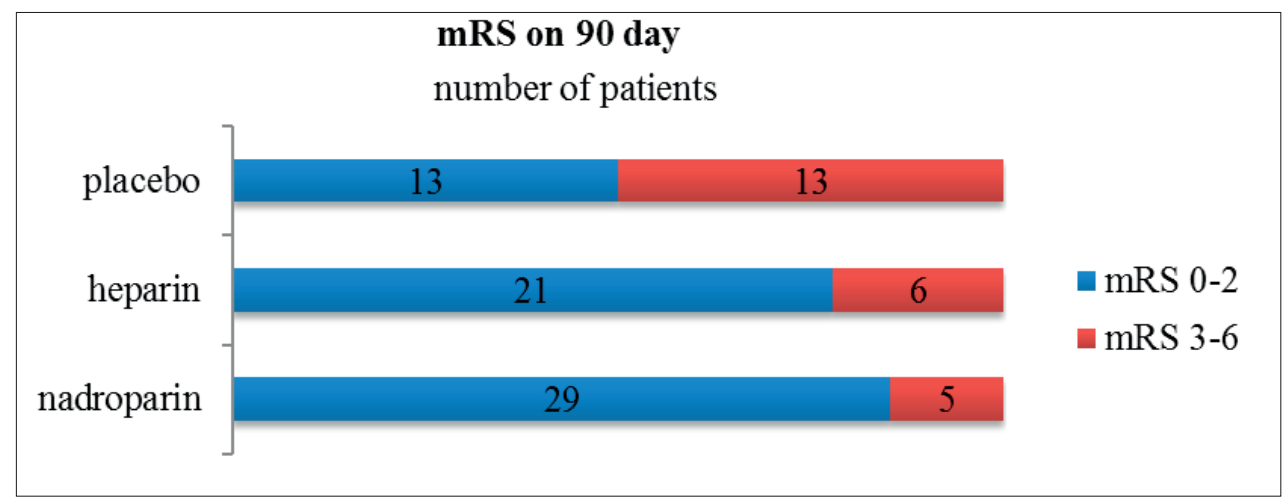

Fig. 3. mRS 0-2 on 90th day.

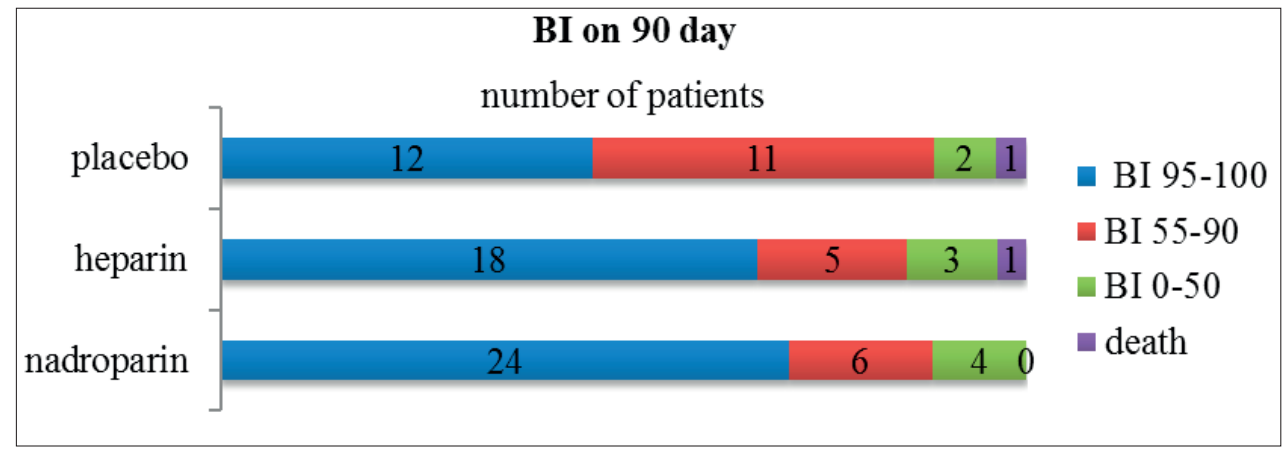

Fig. 4. BI on 90th day. 
that such a designed study would not now be published. For example, in $34 \%$ of all patients enrolled in the IST, the treatment was initiated later than $24 \mathrm{~h}$ after the onset of stroke ${ }^{15}$. In our study, all patients were enrolled within $24 \mathrm{~h}$ after the onset of ischemic stroke. $28 \%$ of the IST patients had uncontrolled arterial hypertension with systolic pressure values over $180 \mathrm{mmHg}$. The systolic blood pressure during the treatment was maintained below $180 \mathrm{mmHg}$. Almost a third of randomized patients were more than 80 years old. Our cohort consisted of patients younger than 80 years old. The most serious limitation of the study is that almost one third of all patients had no CT scan prior to the treatment. In our study, all patients underwent an initial brain CT scan before the beginning of the treatment and a follow-up CT scan $24 \mathrm{~h}$ later. Intracerebral hemorrhage was not detected in any of the patients. These days each of the above mentioned factors would exclude the patient from the reperfusion therapy due to the risk of intracerebral hemorrhage.

The efficacy and safety of heparin compared to placebo administered within $3 \mathrm{~h}$ from the stroke onset was tested in a clinical study published by Camerlingo ${ }^{14}$. The study showed that $38.9 \%$ of heparin treated patients had an $\mathrm{mRS}$ score $0-2$, what was significantly more $(P=0.025)$ than patients receiving placebo $(28.6 \%)$. There were nonsignificant differences in deaths in the groups (heparin $16.8 \%$ vs placebo $21.9 \%, P=0.189$ ). Symptomatic intracerebral hemorrhage occurred significantly more frequently in the heparin group compared with the placebo group (6.2\% vs $1.4 \%, P=0.008)\left(\right.$ ref. $\left.{ }^{14}\right)$.

In our group of patients, we found a statistically significant difference in self-independence $\mathrm{mRS}$ with a score of $0-2$ on the $90^{\text {th }}$ day between the heparin and the placebo groups ( $80 \%$ heparin vs. placebo $50 \%, P=0.0350$ ). During the period of the trial, we recorded two deaths (one $(3.7 \%)$ in the heparin and one death $(3.8 \%)$ in the placebo group). Patients died within the first 30 days. No haemorrhagies (intracebral or systemic) were present. The cause of death in both patients was cardiorespiratory arrest.

Many studies with LMWH are also rather confusing. Moreover, most of were designed as secondary prevention studies not as an acute stroke treatment. Kay et al. 1995 compared the therapeutic dose of nadroparin with a placebo administered within $48 \mathrm{~h}$ in subjects with the acute ischemic stroke. They found that in patients with ischemic stroke treated within $48 \mathrm{~h}$ of the onset of symptoms, nadroparin was not effective in improving outcomes at three months $(P=0.12)$, but demonstrated improvement in outcomes at six months $(P=0.005)$ (ref. $\left.{ }^{16}\right)$. Hemorrhagic transformation occurred in $6.2 \%$ patients treated with nadroparin in the therapeutic dose, $8.6 \%$ in prophylactic nadroparin, and even $12 \%$ in the placebo group $^{16}$. In the FISS-tris study, LMWH or aspirin were administered within $48 \mathrm{~h}$ after the stroke onset. LMWH was significantly associated with reduction of early neurological deterioration within the first 10 days, compared to aspirin (absolute risk reduction, $7.2 \%$; odds ratio [OR], 0.44; 95\% CI, 0.21-0.92) (ref. ${ }^{23}$ ). In our study, there were significant differences in self independence evaluated by $\mathrm{mRS}$ on the $90^{\text {th }}$ day in the nadroparin group compared to the placebo group (29 (85\%) vs. $13(50 \%), P=0.0031)$. In our cohort, we found no systemic or cerebral hemorrhage and no treatment-connected death occured.

Previous studies of the safety profile of heparin and nadroparin reported increased occurrence of serious adverse events such as bleeding complications and mortality associated with treatment. In our study, we did not observe any manifestations of bleeding, either symptomatic or asymptomatic despite the full therapeutic (anticoagulant) dose. We assume that the absence of hemorrhagic complications was due to the strict selection of patients before the treatment based on well-selected inclusion and exclusion criteria. We excluded those patients with signs of large ischaemia on the initial CT scan (more than $1 / 3$ of middle cerebral artery territory), or with complicated severe neurological disability (NIHSS over 25, in fact the highest score was 17). It is difficult to precisely compare treatment efficacy among different clinical trials despite the use of the same rating scales (NIHSS, mRS, BI) as the studies use different variations of the scales, as well as different score intervals.

This is a pilot study with a unique design, focusing on high safety of the used method, which makes it relevant, even though in comparison with other large studies (eg. IST, Camerlingo, FISS) our cohort is small ${ }^{14-15,23}$. Our study allows a direct comparison of the safety and therapeutic effect of nadroparin, heparin and placebo administered in therapeutic doses within $24 \mathrm{~h}$ from the stroke onset.

\section{CONCLUSIONS}

The results of our pilot study show that both heparin and nadroparin treatments in therapeutic doses are safe in comparison to placebo and they are also effective in the acute phase of ischemic stroke. However, it is necessary to confirm our findings with multicentric clinical trials with larger numbers of patients.

\section{ABBREVIATIONS}

aPTT, Activated Partial Thromboplastin Time; ASA/ AHA, The American Heart Association/American Stroke Association; BI, Barthel Index; CT, Computer tomography; ESO, European Stroke Organisation; FISS, Fraxiparin in stroke study for the treatment of ischemic stroke; IST, International Stroke Trial; IVT, Intravenous thrombolysis; LMWH, Low molecular weight heparin; mRS, Modified Rankin Scale; NIHSS, National Institutes of Health Stroke Scale; NINDS, National Institute of Neurological Disorders and Stroke; SD, Standard deviation.

Acknowledgment: Study is supported by projects ITMS:26220220187 "Martin Centre for Biomedicine". 
Author contributions: JD, VN, EK: literature search, study design, data interpretation, data analysis, critical revision, final approval; SS: data interpretation, data analysis, critical revision; RD translation, data analysis; KK, MTK, EK data interpretation, critical revision.

Conflict of interest statement: The authors state that there are no conflicts of interest regarding the publication of this article.

\section{REFERENCES}

1. Bonita R, Stewart A, Beaglehole R. International trends in stroke mortality: 1970-1985. Stroke 1990;21:989-92.

2. Feigin VL, Lawes CMM, Bennett DA, Anderson CS. Stroke epidemiology: a review of population-based studies of incidence, prevalence, and case fatality in the late 20th century. Lancet Neurol 2003;2(1):43-53.

3. Hankey GJ, Warlow C. Treatment and second prevention of stroke: evidence, costs and effects on individuals and populations. Lancet 1999;354(9188):1457-63.

4. Rödén-Jüllig $\AA$, Britton M. Effectiveness of heparin treatment for progressing ischaemic stroke: before and after study. J Intern Med 2000;248(4):287-91.

5. Bejot Y, Rouaud O, Durier J. Decrease in the stroke case fatality rates in a French population-based twenty-year study. Cerebrovasc Dis 2007;24:439-44.

6. Tissue Plasminogen Activator for Acute Ischemic Stroke. The National Institute of Neurological Disorders and Stroke rt-PA Stroke Study Group. N Engl J Med 1995;333(24):1581-7.

7. Hacke W, Kaste M, Bluhmki E. Thrombolysis Alteplase 3 to 4,5 Hours after Acute Ischemic Stroke. N Eng J Med 2008;359(13):1317-29.

8. Berkhemer OA, Fransen PS, Beumer D. A randomized trial of intraarterial treatment for acute ischemic stroke. New England Journal of Medicine 2015;372:11-20.

9. Campbell BCV, Mitchell PT, Kleining TJ. Endovascular Therapy for Ischemic Stroke with Perfusion-Imaging Selection. N Engl J Med 2015;372(11)-1009-18.

10. Goyal M, Demchuk AM, Menon BK. Randomized assessment of rapid endovascular treatment of ischemic stroke. $\mathrm{N}$ Engl J Med 2015;372(11):1019-30.

11. Marsh EE, Adams HP, Biller J, Wasek P, Banwart K, Mitchell V, Woolson R. (1989). Use of antithrombotic drugs in the treatment of acute ischemic stroke A survey of neurologists in practice in the United States. Neurology 1989;39(12):1631-4.

12. Bath P, Leonardi-Bee J, Bath F. Low molecular weight heparin versus aspirin for acute ischemic stroke: a systematic review. J Stroke Cerebrovasc Dis 2002;11(2):55-62.

13. Berge $E$, Abdelnoor M, Nakstad PH, Sandset PM. Low molecularweight heparin versus aspirin in patients with acute ischaemic stroke and atrial fibrillation: a double-blind randomised study: HAEST Study Group: Heparin in Acute Embolic Stroke Trial. Lancet 2000:355(9211):1205-10.

14. Camerlingo M, Salvi P, Belloni G. Intravenous Heparin Started Within the First 3 Hours After Onset of Symptoms as a Treatment for Acute Nonlacunar Hemispheric Cerebral Infrartions. Stroke 2005;36(11):2415-20.

15. International Stroke Trial Collaborative Group. The International Stroke Trial (IST): a randomised trial of aspirin, subcutaneous heparin, both, or neither among 19435 patients with acute ischaemic stroke. Lancet 1997;349(9065):1569-81.

16. Kay R, Wong KS, Yu YL. Low-molecular-weight heparin for the treatment of acute ischemic stroke. N Engl J Med 1995; 333(24):1588-94.

17. Paciaroni M, Agnelli G, Micheli S, Caso V. Efficacy and safety of anticoagulant treatment in acute cardioembolic stroke: a meta-analysis of randomized controlled trials. Stroke 2007;38(2):423-30.

18. Rothwell PM, Coull AJ, Giles MF. Change in stroke incidence, mortality, case-fatality, severity, and risk factors in Oxfordshire, UK from 1981 to 2004 (Oxford Vascular Study). Lancet 2004;363(9425):192533.

19. Sandercock PA, Counsell C, Kamal AK. Anticoagulants for acute ischeaemic stroke. Cochrane Database Syst Rev 2008;(4):CD000024.

20. European Stroke Organisation (ESO) Executive Committee, and ESO Writing Committee. Guidelines for management of ischaemic stroke and transient ischaemic attack 2008. Cerebrovasc Dis 2008;25(5):457-507.

21. Jauch EC, Saver JL, Adams HP. Guidelines for the early management of patients with acute ischemic stroke a guideline for healthcare professionals from the American Heart Association/American Stroke Association. Stroke 2013;44(3):870-947.

22. The Publications Committee for the Trial of ORG 10172 in Acute Stroke Treatment (TOAST) Investigators. Low Molecular Weight Heparinoid, ORG 10172 (Danaparoid), and Outcome After Acute Ischemic Stroke: A Randomized Controlled Trial. JAMA 1998;279(16):1265-72.

23. Wang Q, Chen C, Chen XY. Low-molecular-weight heparin and early neurologic deterioration in acute stroke caused by large artery occlusive disease. Arch Neurol 2012; 69(11):1454-60. 